\title{
Estadística descriptiva: identificar, medir, almacenar, organizar y resumir la información
}

\author{
David Santiago-Germán* \\ Servicio de Urgencias, Hospital General Regional N.० 1 «Dr. Carlos Mac Gregor Sánchez Navarro», IMSS, Ciudad de México, México
}

\begin{abstract}
Resumen
Diariamente recibimos grandes cantidades de información a través de nuestros sentidos, información que es recopilada, almacenada, analizada y transformada por nuestro cerebro para la solución de problemas. La estadística es la ciencia de recolectar, organizar, resumir y analizar información para responder preguntas y formular conclusiones. Esta rama de la ciencia es inherente a nuestra vida profesional cotidiana, y comprenderla puede transformar nuestra forma de ver, pensar $y$ actuar de una práctica basada en la experiencia a la práctica basada en la evidencia. Éste es el primero de varios artículos que tienen como objetivo que el lector comprenda conceptos básicos de estadística médica que le permitan interpretar un documento científico de investigación. En este artículo se explican los principios básicos de la estadística descriptiva: variables, unidad de medida, escalas de medida y medidas de resumen (medidas de tendencia central, dispersión y posición).
\end{abstract}

Palabras clave: Estadística descriptiva. Variables.

\section{Descriptive statistics: identifying, measuring, storing, organizing, and summarizing the information}

\begin{abstract}
Daily we receive large amounts of information through our senses, this is compiled, storaged, analyzed, and transformed by our brain to solve problems. Statistics is the science of gathering, organizing, summarizing, and analyzing information to answer questions and formulate conclusions. This branch of science is inherent to our professional life, its understanding can transform our way of seeing, thinking, and acting, from an practice based on experience into one based on evidence. This is the first of various articles whose goal is to make the reader familiar with medical statistics allowing him to manage easily a scientific research document. This paper explains the basic principles of descriptive statistics, variables, unit of large amounts measurement scales, and summary measures.
\end{abstract}

Key words: Descriptive statistics. Variables.

\section{Correspondencia:}

*David Santiago-Germán

Gabriel Mancera, 222

Col. Del Valle

Fecha de recepción: 09-01-2019

Fecha de aceptación: 19-01-2019

DOI: 10.24875/REIE.M19000006
Disponible en internet: 08-02-2019 Rev Educ Investig Emer. 2019;1(1):32-36 www.medicinadeemergencias.com do por Permanyer México SA de CV. Este es un artículo Open Access bajo la licencia CC BY-NC-ND (http://creativecommons.org/licenses/by-nc-nd/4.0/). 
Todos los días nuestro cerebro recibe y procesa grandes cantidades de información. Cuando atendemos a un enfermo, en el consultorio o en el área de choque, recopilamos información a través del interrogatorio, la exploración física y los estudios de laboratorio y gabinete, con la finalidad de llegar a una conclusión diagnóstica que nos permita elegir el mejor tratamiento y predecir el desenlace. Por medio del interrogatorio, a través de la comunicación verbal y no verbal, recolectamos información del paciente. Formular la pregunta correcta puede enfocar nuestra atención hacia la potencial causa del problema o, por el contrario, una pregunta incorrecta puede desviarnos totalmente y conducirnos a un diagnóstico erróneo, con la consecuente falla terapéutica y desenlace fatal.

Un buen médico no es aquél que cree tener todas las respuestas, sino aquél que hace las preguntas correctas. $^{1}$

Durante la exploración física recolectamos información a través de los órganos de los sentidos y los instrumentos colocados a la cabecera del paciente como monitores de la actividad eléctrica cardíaca, esfingomanómetros, oximetría de pulso, capnógrafos, termómetros, glucómetros, ultrasonido, etc. Por último, empleamos métodos de análisis moleculares, de tejidos y de imagen cada vez más sofisticados y precisos. La utilidad y calidad de la información recopilada dependerá entonces de la atención, interés o curiosidad con la que observemos, de la eficacia en la comunicación o relación médico-paciente y del grado de precisión de los instrumentos de medición. A través de los años, nuestro cerebro ha aprendido a reconocer y asociar patrones del proceso salud-enfermedad, e intuitivamente formulamos hipótesis que llamamos diagnósticos y las sometemos a prueba al iniciar un tratamiento.

Un buen médico es aquél que presta atención y, con los instrumentos de medición que tiene a su alcance, recolecta la mayor cantidad de información, la ordena e identifica el problema.

Diariamente recopilamos y analizamos información de nuestros pacientes, y generamos conocimiento basado en la experiencia que adquirimos con el paso de los años para transferirlo de tutor a alumno por generaciones. En la era moderna, la tecnología de la información nos permite almacenar datos en cantidades casi inimaginables en pequeños espacios físicos y transferirlos a largas distancias a gran velocidad. ¿Qué hacemos con estos datos?, ¿cómo se analizan?, ¿cómo se transforman los datos en respuestas a los problemas médicos?, ¿cómo debemos interpretarlos? y ¿cómo se verifica la veracidad del conocimiento generado? Para responder a estas y otras preguntas utilizamos la estadística.

\section{¿Qué es la estadística?}

La estadística es la ciencia de recolectar, organizar, resumir y analizar información para responder preguntas y formular conclusiones. ${ }^{2}$

En el escenario médico la información consiste en síntomas, signos, medidas físicas (como el color de la piel, la estatura, el peso, la temperatura) y bioquímicas (como la concentración de colesterol y triglicéridos en la sangre). Estas características que poseen todos los individuos y que los hacen únicos se conocen como variables. Una variable es una característica de un fenómeno, objeto o individuo. Toda variable se puede medir, es decir, se le puede asignar un valor numérico que represente su magnitud física. La cantidad estandarizada de una determinada magnitud física se conoce como unidad de medida; por ejemplo, los segundos (s), minutos (min), horas (h) y años son unidades de medida de la variable tiempo. No obstante, no todas las variables poseen una unidad de medida estandarizada, y en algunas se utilizan etiquetas o categorías para definir un atributo en un individuo. De acuerdo a la unidad de medida empleada, las escalas de medición de las variables son:

- Escala numérica: se asigna un valor numérico, haciendo posible la realización de operaciones aritméticas.

- Escala categórica: se asignan nombres a cada categoría.

Las variables numéricas o cuantitativas se clasifican en discretas y continuas. Se llaman discretas si su valor se representa en números finitos o enteros y continuas si su valor se representa en números infinitos.

De acuerdo a su escala de medición, las variables categóricas o cualitativas se clasifican en nominales, ordinales, de intervalo y de razón. ${ }^{3}$ Una variable es nominal si cuenta con varias categorías sin un orden natural, como, por ejemplo, los grupos sanguíneos A, B, AB, O. Si la variable nominal tiene sólo dos posibles categorías que indiquen la presencia o ausencia de una característica, se denomina dicotómica, como, por ejemplo, el factor Rh de una persona (dato nominal no numérico) o el código postal (dato nominal numérico). Una variable es ordinal si cuenta con varias categorías con un orden natural o jerárquico pero unidades de medida no definidas, como, por ejemplo, la escala de Daniels para la evaluación de la fuerza muscular (dato ordinal numérico). Una escala de medición ordinal con intervalos entre cada categoría bien definidos por una unidad de medición fija se conoce como variable 


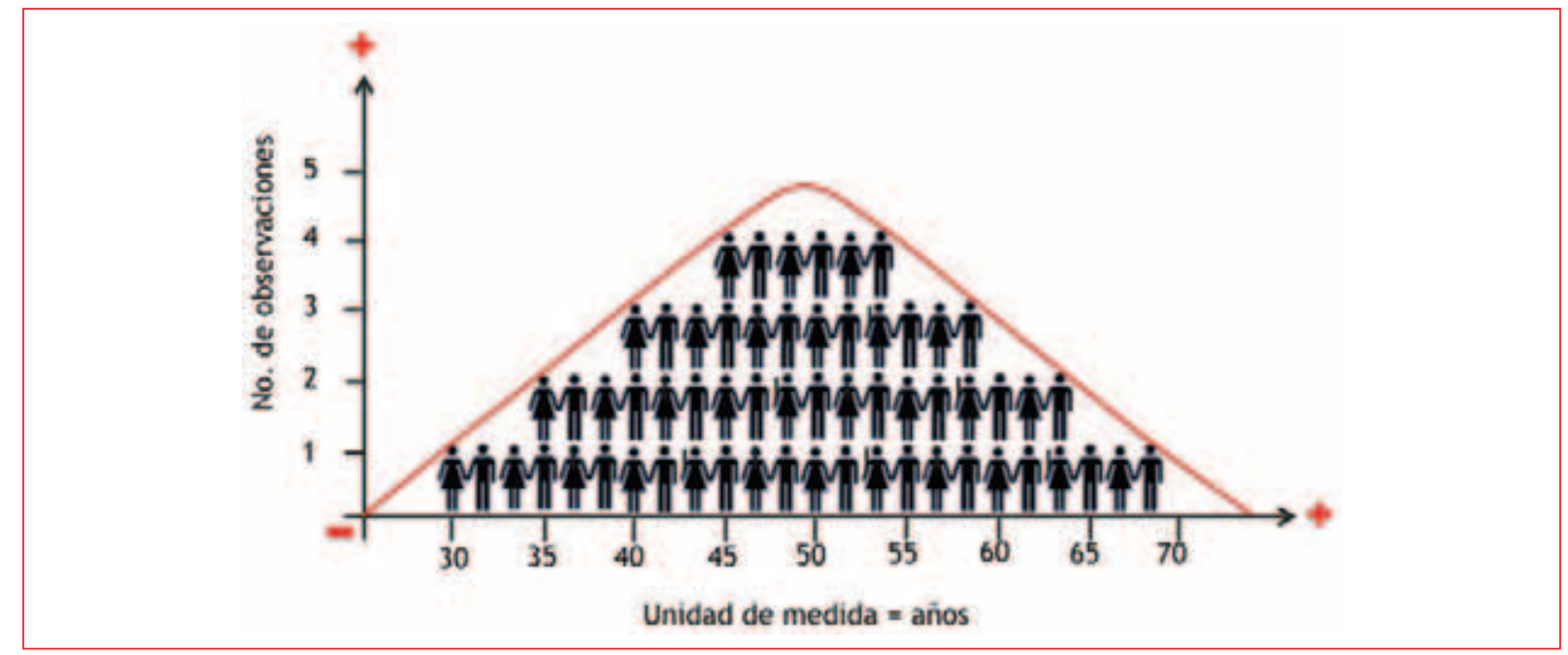

Figura 1. Curva de distribución de la variable edad en una muestra de 60 individuos. En el eje de las abscisas ( $\mathrm{x}$ ) se indica la unidad de medida en años y en el de las ordenadas (y), el número de observaciones. Los valores se han ordenado de menor a mayor y algunos de ellos se repiten más de una vez formando una curva convexa.

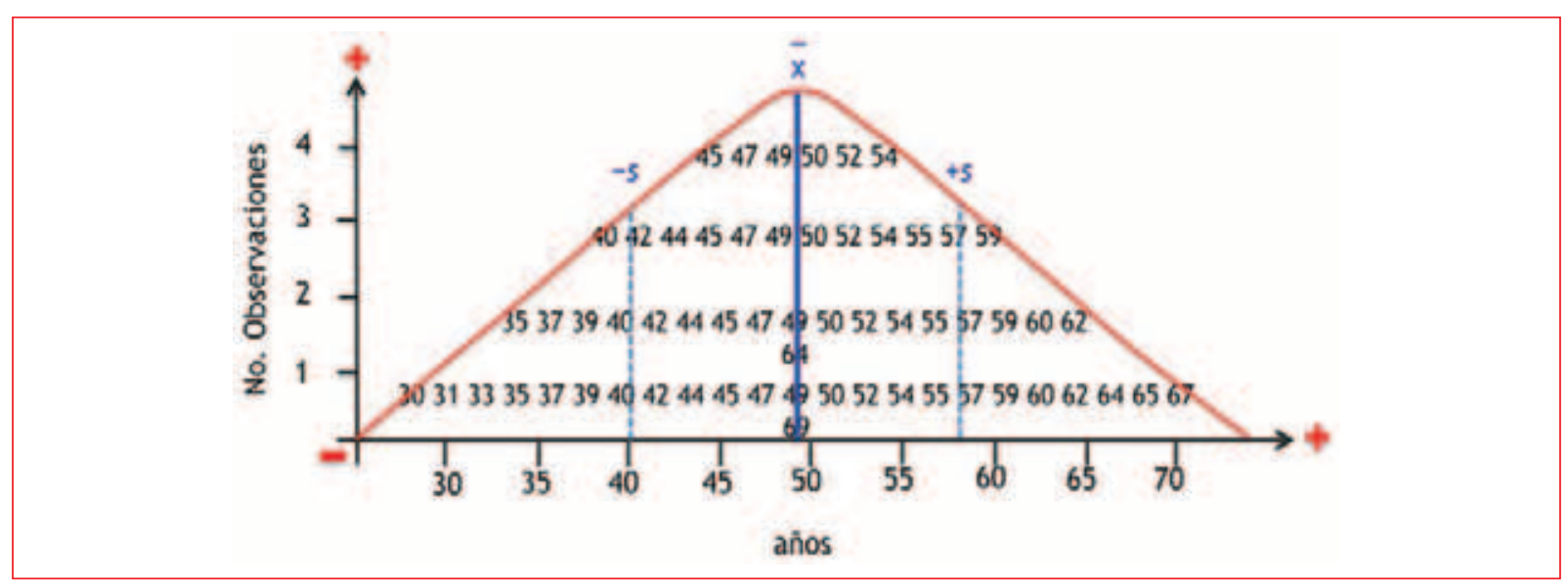

Figura 2. Medidas de dispersión: desviación estándar. Es el promedio de las distancias entre cada valor y la media. $\mathrm{x}$ : media; s: del inglés standard deviation.

de intervalo, como, por ejemplo, la escala de temperatura en grados Celsius $\left({ }^{\circ} \mathrm{C}\right)$, que divide en 100 grados el intervalo comprendido entre el punto de congelación $\left(0^{\circ} \mathrm{C}\right.$ o cero relativo) y el punto de ebullición $\left(100^{\circ} \mathrm{C}\right)$ del agua. Por último, una variable de razón es aquella variable de intervalo que comienza desde el cero y aumenta en intervalos sucesivos iguales, como, por ejemplo, la escala de temperatura en grados Kelvin $\left({ }^{\circ} \mathrm{K}\right)$, donde $0^{\circ} \mathrm{K}$ o cero absoluto representa la ausencia de energía que emite un cuerpo por la ausencia de movimiento de sus moléculas.

Un individuo posee más de una variable, e identificarlas puede ser difícil. El conjunto de variables de un grupo de individuos puede parecer complejo a simple vista, pero una vez que se ordena y se resume se ve más claro.

\section{¿Qué es la estadística descriptiva?}

Consiste en organizar y resumir la información recolectada. Cuando medimos la misma variable en un grupo de individuos, obtenemos un conjunto de valores. Para representar un conjunto de datos hay que ordenar y resumir sus valores (Fig. 1). Una curva de distribución es una representación gráfica de los valores de una variable de un conjunto de individuos, ordenados de menor a mayor en un plano cartesiano. ${ }^{1}$ 


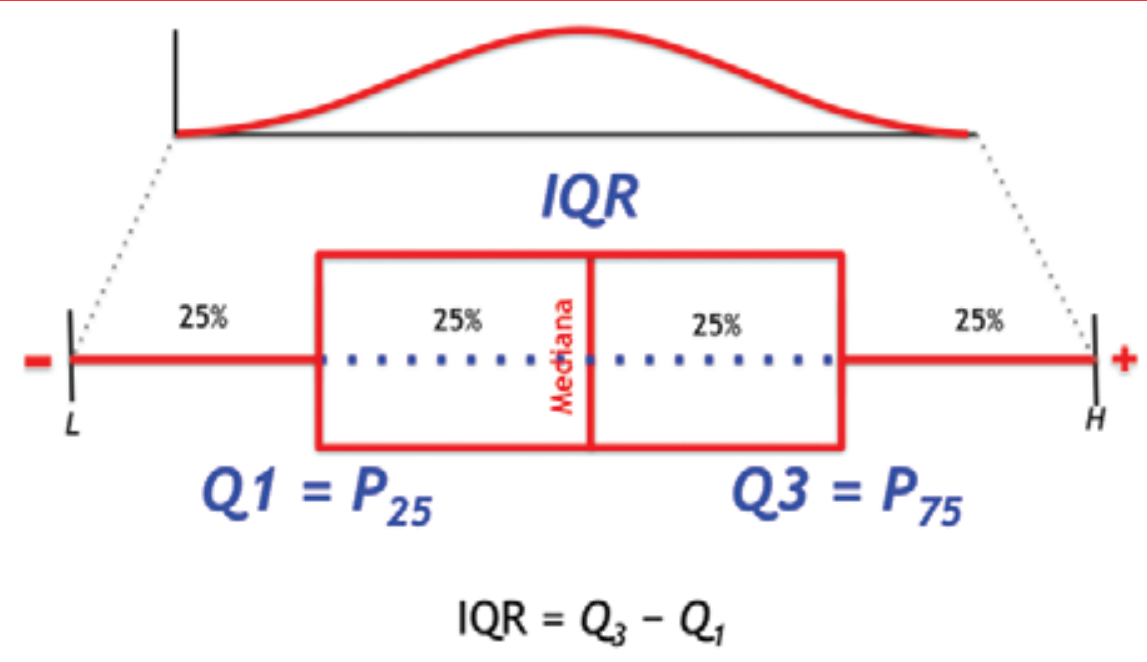

Figura 3. Medidas de dispersión: rango intercuartílico. Se obtiene después de ordenar los valores de menor a mayor y restar el valor ubicado en el percentil 25 al valor ubicado en el percentil 75.

$\mathrm{P}_{25}$ : percentil 25; $\mathrm{P}_{75}$ : percentil 75 .

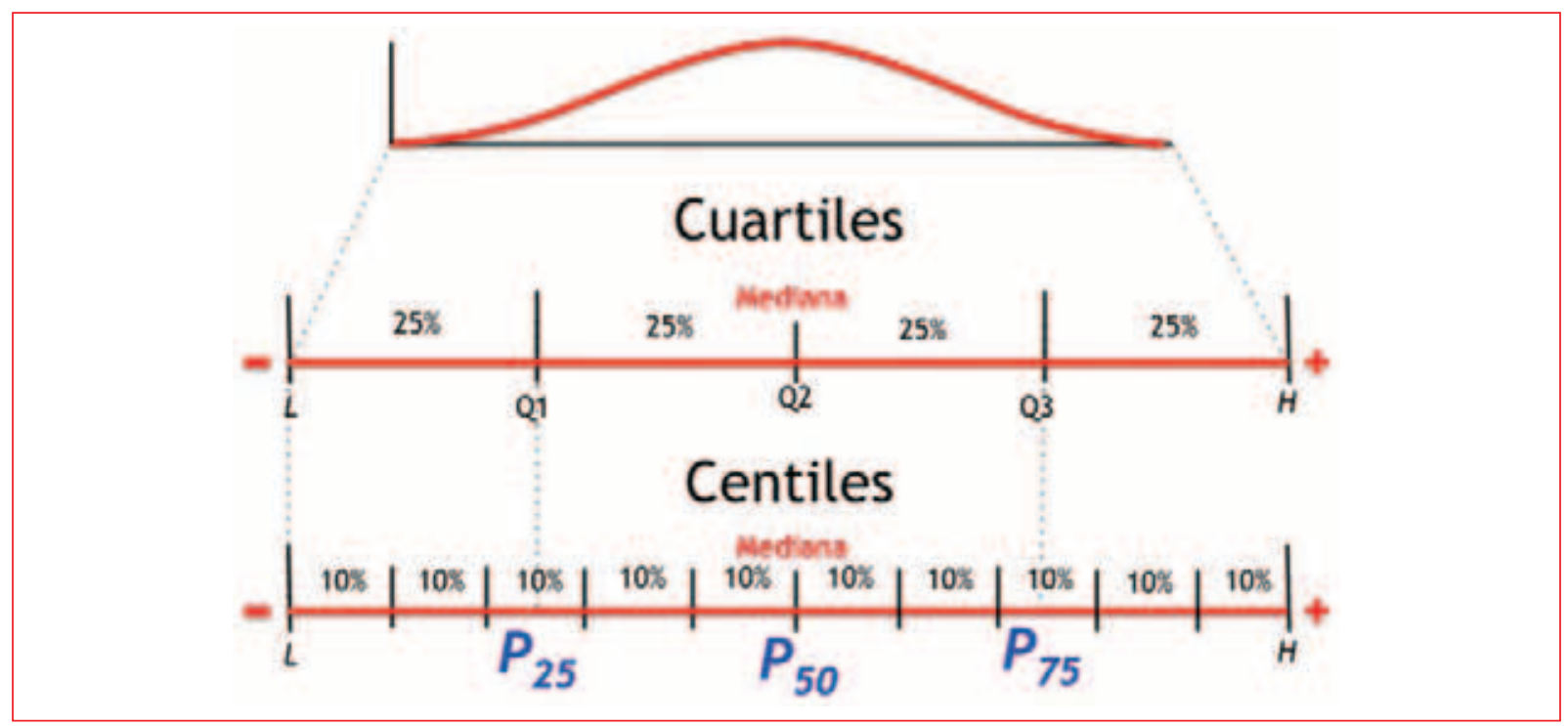

Figura 4. Medidas de posición. Después de ordenar los valores de menor a mayor, se divide en cuatro o en cien partes iguales para obtener los cuartiles y los percentiles, respectivamente.

$\mathrm{L}$ : dato de menor valor; $\mathrm{H}$ : dato de mayor valor; 01: primer cuartil; 02: segundo cuartil; Q3: tercer cuartil;

$\mathrm{P}_{25}$ : percentil 25; $\mathrm{P}_{50}$ : percentil 50; $\mathrm{P}_{75}$ : percentil 75.

\section{Medidas de tendencia central o promedios}

Los valores numéricos que se localizan en la parte central de una curva de distribución de un conjunto de datos se llaman medidas de tendencia central o promedios. La media es la suma de todos los valores dividida entre el número de observaciones. La mediana es el valor que se localiza a la mitad después de haber ordenado todos los valores de menor a mayor. Y la moda es el valor más frecuente. ${ }^{4,5}$

\section{Medidas de dispersión}

Son valores numéricos que describen el grado de dispersión, separación o variabilidad de los datos. La desviación estándar es el promedio de las distancias entre cada valor y la media (Fig. 2). El rango o amplitud 
es la diferencia entre el dato de mayor valor y el de menor valor. El rango intercuartílico (interquartile range [IQR]) es la diferencia entre el percentil 75 y el 25 (Fig. 3). 4,5

\section{Medidas de posición}

Son valores numéricos que localizan un dato específico en relación con el resto de la muestra (Fig. 4). Los cuartiles son números que dividen un conjunto de datos ordenados en cuatro partes iguales. Los centiles o percentiles son números que dividen un conjunto de datos ordenados en 100 partes. $^{5}$

Éste es un primer acercamiento a la estadística aplicada a la medicina. Identificar, medir, recopilar, organizar y resumir información maximizará nuestra comprensión del proceso salud-enfermedad de nuestros pacientes. La estadística es inherente a la práctica médica cotidiana, y comprenderla transformará nuestra práctica basada en la experiencia en una práctica basada en la evidencia.

\section{Bibliografía}

1. Martínez González A, Sánchez-Mendiola M. La pregunta de investigación en educación médica. Inv Ed Med. 2015;4(13):42-9.

2. Hulley SB, et al. Designing Clinical Research. $4 .^{\text {a }}$ ed. Filadelfia: Editorial Wolters Kluwer/Lippincott Williams \& Wilkins; 2013.

3. Carney S, Doll H. Introduction to biostatistics: Part 1. Measurement scales and their summary statistics. ACP J Club. 2005;143(1):A8-9.

4. Whitley E, Ball J. Statistics review 1: presenting and summarizing data. Crit Care. 2002;6(1):66-71

5. Dawson G. Interpretación fácil de la bioestadística: la conexión entre la evidencia y las decisiones médicas. 1. ${ }^{\text {a }}$ ed. España: Editorial Elsevier; 2009. 